\title{
CMB Anisotropies in the Presence of Extra Dimensions
}

\author{
C. S. Rhodes ${ }^{1}$, C. van de Bruck ${ }^{2}$, Ph. Brax ${ }^{3}$ and A.-C. Davis ${ }^{1}$ \\ ${ }^{1}$ Department of Applied Mathematics and Theoretical Physics, Centre for Mathematical Sciences, University of Cambridge \\ Wilberforce Road, Cambridge CB3 OWA, U.K. \\ 2 Astrophysics Department, Oxford University, Keble Road \\ Oxford OX1 3RH, U.K. \\ 3 Service de Physique Theorique, CEA-Saclay \\ F-91191 Gif/Yvette Cedex, France
}

\begin{abstract}
We discuss the effect of the time evolution of extra dimensions on CMB anisotropies and largescale structure formation. We study the impact of scalar fields in a low-energy effective description of a general class of brane world models on the temperature anisotropy power spectrum. We show that when the coupling between these scalar fields and matter evolves over cosmological timescales, current observations of the $\mathrm{CMB}$ anisotropies can constrain primordial values of the fields in a manner complementary to local, late-time tests of gravity. We also present the effect of these fields on the polarization anisotropy spectra and the growth of large-scale structure, showing that future CMB observations will constrain theories of the Universe involving extra dimensions even further.
\end{abstract}

PACS-Nr.: 98.80.-k, 98.80.Es, 04.50.+h, 11.25.Wx

\section{INTRODUCTION}

The dominant force in the universe is gravity. According to modern cosmological theories, primordial density perturbations were generated in the very early universe by quantum fluctuations, whereas gravity shaped the structures of the universe [1]- [3]. This standard picture of structure formation by gravitational instability has gained remarkable support from observations of the fluctuations in the cosmic microwave background (CMB) radiation [4]. In addition, predictions of hierarchical clustering on scales up to $1000 \mathrm{Mpc}$ in the context of cold dark matter theories (CDM) are in very good agreement with observations of the large scale structures in the universe. These observations may be used to determine the present cosmological parameters, such as the density of cold dark matter, the age of the universe and the equation of state of dark energy among others.

These observations allow us not only to determine cosmological parameters, but also to test our theories of gravity itself. So far, no observation of a deviation from General Relativity (GR) has been reported, but, as we shall see, observations in the CMB and the large scale structures (LSS) provide important complementary tests to experiments on Earth or in the Solar System which constrain deviations from GR. The growth of perturbations predicted by a given theory of gravity is sensitive to the details of the theory; therefore, the study of perturbations can give important insights about any deviations from GR at different cosmological epochs.

One well-known class of models where deviations from GR are predicted is that of scalar-tensor theories, in which the gravitational sector contains not only a tensor field but also scalar fields. The Brans-Dicke theory is an example of such a scalar-tensor theory (see [5] for a recent review on scalar-tensor theories). One particular feature of these models is that Newton's constant is no longer constant in the cosmological evolution; alternatively, in the so-called Einstein frame, which can be obtained from the original theory after a conformal transformation and field redefinitions, Newton's constant is truly constant, but the masses of particles are no longer independent of the spacetime coordinates. Calculations of CMB anisotropies in scalar-tensor theories [6]- [8] show that the positions and amplitudes of the acoustic peaks usually depend on the parameters of the theory. There is no general trend on how the peaks are affected, because the dynamics of the fluctuations depend critically on the evolution of the scalar field and its coupling to matter; but the positions and amplitudes of all peaks are affected. Similarly, the slope and amplitude of the matter power spectrum are affected. In [6], [7] and [8] certain models of scalar-tensor theories and their cosmological consequences are discussed in considerable depth. In [9] and [10] models are discussed in which the scalar field plays the role of dark energy and couples to dark matter. In [11] it is shown that CMB anisotropies can probe deviations from the standard Friedmann equation.

The studies of scalar-tensor theories and their cosmological consequences are of considerable interest, because theories beyond the Standard Model of particle physics imply that GR is not fundamental. Prime examples of theories in which deviations from GR are expected are those involving supergravity and superstrings (or their extension: Mtheory) [12]. In particular, models which predict the existence of extra dimensions can at low energies usually be described as scalar-tensor theories. Typically, the low energy effective description of higher dimensional theories contains many light scalar fields (so-called moduli fields), which couple to matter in a manner dependent on the details of the higher dimensional theory. In this paper we shall discuss the scalar fields appearing in brane world 
models and their effect on the evolution of cosmological perturbations (for recent reviews on brane worlds see, for instance, [13]- [15] and references therein). The brane world model we consider is of the two brane kind. The model is quite generic and contains the well-known Randall-Sundrum brane world model (RS I) [16] as a special case. As it turns out (see section II below), the model predicts the existence of two scalar fields at low energies. One of the scalar degrees of freedom is associated with a bulk scalar field, i.e. a scalar field which can propagate in the higher dimensional spacetime between the branes. The other scalar degree of freedom is related to the physical distance between the branes. The two scalar fields couple to matter differently and thereby affect the growth of perturbations in the universe each in their own way.

In the theory to be discussed, the coupling function of one of the scalar fields is constant, its value depending on only one free parameter of the higher-dimensional theory. This parameter has to be chosen to be small in order for the theory to be consistent with local (Solar System) experiments. The other coupling function, however, depends on the second field itself. Barring any stabilising mechanism, this field evolves in time due to the cosmological evolution. Thus, even though the coupling must be small today, it could have been larger in the early universe - in particular at the epoch of matter-radiation decoupling. Interestingly, in our theory the matter coupling of the second field is driven toward small values during the matter dominated epoch, meaning that the theory can easily be made consistent with experiments in the solar system ${ }^{1}$.

In this paper we discuss the impact of the moduli fields on the evolution of cosmological perturbations. We compare the predictions to the $\Lambda \mathrm{CDM}$ model. We furthermore assume that there is no potential energy for the scalar fields, so that the energy density of the scalar field is always much smaller than radiation or matter energy density. In the models used in this paper, the reported acceleration of the universe at low redshift is always caused by a cosmological constant. This ensures that the distance to the last scattering surface and the time of matter-radiation equality is only marginally affected by the presence of the scalar fields. Furthermore, in order to distinguish between the traces of the different fields we study them individually.

The paper is organised as follows: In section II we briefly review the five dimensional setup of a brane world theory and discuss the effective theory in four dimensions; readers familiar with brane worlds or not interested in the details may skip this part. In section III we write down the action to be discussed in this paper and derive the field equations. We also study the cosmological background evolution of the fields. In section IV we derive the perturbation equations and discuss the influence of the individual fields on CMB anisotropies and the matter power spectrum, and we conclude in section V. This paper is addressed to readers with different backgrounds, so we have attempted to be comprehensive, and have included some pedagogical explanations to highlight salient points; interested readers should of course consult the various cited review articles for further clarification and discussion.

\section{A FIVE DIMENSIONAL THEORY AND ITS EFFECTIVE FIELD THEORY AT LOW ENERGIES}

As an introduction to the theory studied in this paper, we begin with a discussion of a higher-dimensional theory motivated from brane worlds. In these kind of theories, the standard model particles (and maybe also some other, exotic, form of matter) are confined on a four ${ }^{2}$-dimensional object (called a brane), which moves in a higher-dimensional space (known as the bulk). The brane itself carries some intrinsic tension as well. In our model, the bulk spacetime is five-dimensional. This setup is well motivated by recent developments in string theory and provides a useful starting point for more complicated models [18]. Also extracted from string theory is the fact that there is a second brane somewhere in the bulk. Confined on this second brane, which has an intrinsic tension too, can be some form of matter whose only direct interactions with the matter on first brane are mediated via gravity. This matter is a potential dark matter candidate.

The physical spacetime stretches between the two branes, so that the branes form the boundary of the fivedimensional spacetime. The space between is not empty; in general, some form of matter is expected to propagate through the bulk: in the model under consideration, a scalar field is present. Taking this scalar field into account, the overall model setup is presented in figure 1 .

\footnotetext{
${ }^{1}$ Attractor-like behaviour has been found in other scalar-tensor theories as well [17].

${ }^{2}$ Three space and one time.
} 


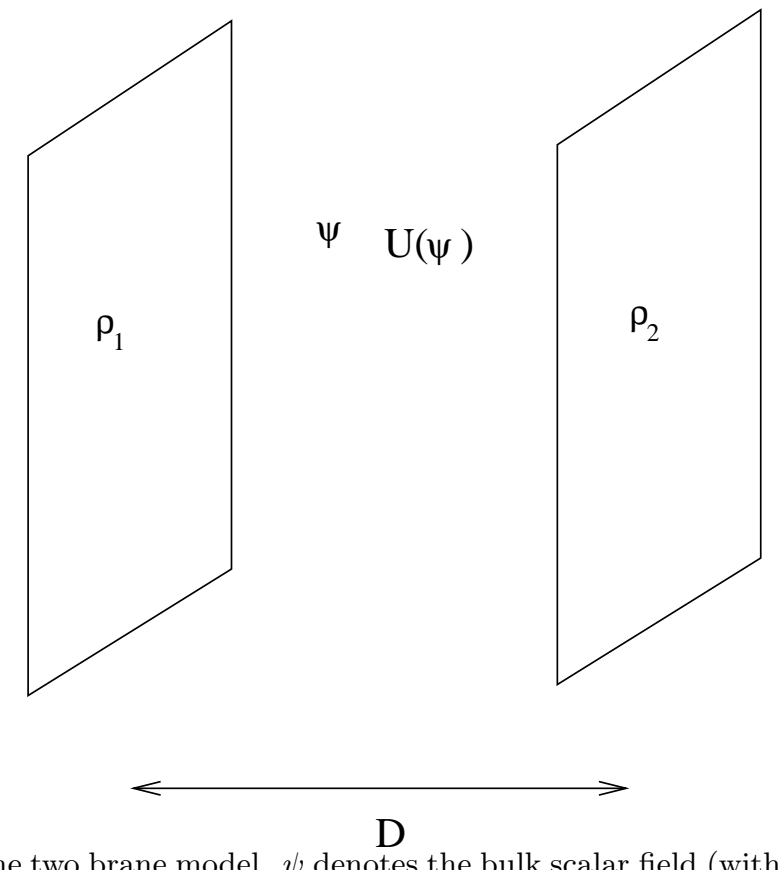

FIG. 1. The setup of the two brane model. $\psi$ denotes the bulk scalar field (with potential energy $U(\psi)$ ), which can propagate between the branes. $\rho_{1}$ and $\rho_{2}$ denote the total (i.e. tension + matter) energy density confined on the individual branes. $D$ is the physical distance between the branes.

For energy densities much higher than the brane tension it has been noted [19] that the expansion of the four dimensional brane universe is not governed by the usual four-dimensional Einstein equations, according to which the expansion rate $H$ is proportional to the matter density $\rho$; instead, it was found that the dependence of $H$ on $\rho$ is $H \propto \rho$. This behaviour was observed in one-brane scenarios [19] but was shown to hold in two-brane scenarios as well (see in particular [20] and [21]). However, for densities much less than the brane tension, the usual law $H \propto \sqrt{\rho}$ can be recovered, albeit with some corrections coming from the scalar fields associated with the bulk dynamics.

As already mentioned in the introduction, the effective field theory at low energies (i.e. at energies much less than the brane tension) can be written as a scalar-tensor theory with two scalar fields. The action of the theory was derived in [22] and reads

$$
S_{\mathrm{EF}}=\frac{1}{16 \pi G_{N}} \int d^{4} x \sqrt{-g}\left[\mathcal{R}-\frac{12 \tilde{\alpha}^{2}}{1+2 \tilde{\alpha}^{2}}(\partial \varphi)^{2}-\frac{6}{2 \tilde{\alpha}^{2}+1}(\partial R)^{2}-V(\varphi, R)\right] .
$$

In this equation, $\mathcal{R}$ is the Ricci scalar, $\varphi$ and $R$ are the two scalar fields and $V$ is the total potential energy of the fields. $\tilde{\alpha}$ is a parameter of the higher-dimensional theory, which will be left free, though note that models of particle physics, in general, will make predictions about its value [23]. In the Einstein frame, each individual matter form on the branes usually couples to the fields $\varphi$ and $R$, so that the action has the general form

$$
S_{\text {Matter }}=S_{\text {Matter }, 1}\left(\psi_{1}, A(\varphi, R) g_{\mu \nu}\right)+S_{\text {Matter }, 2}\left(\psi_{2}, B(\varphi, R) g_{\mu \nu}\right)
$$

where $\psi_{1}$ denotes the matter form on brane 1 and $\psi_{2}$ the matter form on brane 2 . The functions $A$ and $B$ in general depend on both fields and are not equal.

The interpretation of $\varphi$ and $R$ is not straightforward, but the fact that there must be two scalar degrees of freedom at low energies can be understood as follows: first, the bulk scalar field can propagate along the brane directions, because it depends on all spacetime coordinates. The other degree of freedom which appears in the low energy effective action is related to the distance between the branes, which is a scalar function of the four spacetime coordinates. We note furthermore that the case where $\tilde{\alpha}$ is zero (corresponding to the second, field-dependent coupling we consider below) subsumes the Randall-Sundrum two brane model, where there is no bulk scalar field.

The matter couplings are determined by the functions $A$ and $B$, or, to be more precise, by their derivatives with respect to $\varphi$ and $R$. For the brane world model we consider, the coupling functions are given by (see [22] for details)

$$
\begin{aligned}
& \beta_{\varphi}^{(1)} \equiv \frac{\partial \ln A}{\partial \varphi}=-\frac{2 \tilde{\alpha}^{2}}{1+2 \tilde{\alpha}^{2}}, \quad \beta_{\varphi}^{(2)} \equiv \frac{\partial \ln B}{\partial \varphi}=-\frac{2 \tilde{\alpha}^{2}}{1+2 \tilde{\alpha}^{2}}, \\
& \beta_{R}^{(1)} \equiv \frac{\partial \ln A}{\partial R}=\frac{\tanh R}{1+2 \tilde{\alpha}^{2}}, \quad \beta_{R}^{(2)} \equiv \frac{\partial \ln B}{\partial R}=\frac{(\tanh R)^{-1}}{1+2 \tilde{\alpha}^{2}} .
\end{aligned}
$$


The functions $\beta$ depend on the details of the higher dimensional theory, as they provide all information about the nature of the higher-dimensional space (such as its geometry or curvature) relevant at low-energy. Throughout this paper we shall consider coupling functions of the form above.

There are different ways of obtaining the effective theory at low energies. In [22], the moduli space approximation was used, in which one integrates the heavy Kaluza-Klein like excitations out. Strictly speaking, the action above is only valid if the Kaluza-Klein excitations, corresponding to massive particles in four dimensions, are negligible. For example, massive partners of the gravitational zero mode, described by $g_{\mu \nu}$, are neglected. Another method to derive the low-energy effective action was used in [24]- [27], which is based on solving the five-dimensional Einstein equation to linear order, and then projecting onto the brane on which the standard model particles are confined. The theory is then written in the Jordan frame. This method is widely used in works on brane cosmology and has the advantage in that it provides a geometrical framework for the full five-dimensional problem ${ }^{3}$. However, it should be noted that the action with the two methods lead to the same low-energy effective theory: after a transformation to the Einstein frame, the action obtained by the projective approach is equivalent to the action (1).

In practical terms, the use of the moduli space approximation limits the backwards extrapolation in time; for the use of the moduli space approximation to be valid, we require that the Hubble scale be much greater than the scale under consideration. So, $H(z) \approx(1+z)^{2} H_{0}$ must be much less than $k^{-1}$, the size of the extra dimension, so we can see for instance that millimetre (about $10^{13} \mathrm{GeV}^{-1}$ ) scales can be dealt with using this approximation to redshifts of about $10^{15}$ (while numerical integration, of which more discussion in section IV, typically starts at redshifts of $10^{9}$ ). As already said, in deriving the action above, one assumes that Kaluza-Klein excitations are negligible at these redshifts. For example, the bulk scalar field in this approximation does not depend on the extra dimension. We further assume that such excitations are not produced at the epochs which we are interested in. This should be valid because any excitation corresponds to a heavy particle.

Clearly this theory, described by the action in equation (1), is different from GR, and therefore gravitational experiments constrain parameters of the theory. In order for the theory to agree with experiments on earth and observations in the solar system, the parameter $\tilde{\alpha}$ has to be rather small $\left(\tilde{\alpha} \leq 10^{-2}\right)$ and the field $R$ today, should be small $\left(R \leq 10^{-2}\right)$, too. However, whereas the parameter $\tilde{\alpha}$ has to be chosen small from the beginning, the field $R$ is dynamical and so one has to choose its initial condition such that it is small today. Given this, there is another, more intriguing possibility: that during the cosmological history the field is driven towards small values. The cosmological evolution of the system was discussed in [22], where it was shown that $R$ is indeed driven toward small values in a stable fashion. Apart from local experiments, the theory is constrained by cosmological considerations. Firstly, nucleosynthesis bounds have to be respected, which put constraints on both $\tilde{\alpha}$ and the initial value of the field $R$. It is beyond the scope of the paper to constrain the theory by nucleosynthesis as a detailed analysis with two fields involved has to be performed. The second important cosmological constraint on the fields is enforced by CMB anisotropies and large scale structure considerations. We will in this paper develop an understanding of the how the CMB is influenced by the fields.

Before we discuss perturbations in models of the form above, we comment on the frame to be used. The issue of the frame and its physical relevance is not new, and has been hotly discussed in the literature (see e.g. [30]). The reason for choosing the Einstein frame instead of the Jordan frame is that, written in the Jordan frame, one the of the scalar fields has a negative kinetic energy [22] (the metric in the Jordan frame is the induced metric of the first brane, where the standard model particles live). However, this is not a signal of an instability, because in the Einstein frame the kinetic term is positive. The fact that the energy density of the scalar field is not positive definite in the Jordan frame is not unique to field theories derived from brane worlds but appears often in scalar-tensor theories. Several authors have used this as an argument for using the Einstein frame as the physical frame (which is unique), and we follow this argument here. We would like to point out, however, that in the case of the field with non-constant coupling, the matter coupling of this field is very small today, so that at the present epoch the theory is indistinguishable from General Relativity.

\section{COSMOLOGY AND MODULI FIELDS}

As discussed in the last section, there are usually several scalar fields in the low-energy effective action, each of which couples to matter. The goal of this paper is to understand the effects of the individual fields on the evolution

\footnotetext{
${ }^{3}$ In Ref. [28] this formalism was used to calculate the CMB anisotropies in the one-brane scenario of Randall and Sundrum [29].
} 
of perturbations. We shall henceforth discuss them separately, dealing with only one scalar field at a time. We shall see in the next section that there are differences in the predicted CMB power spectrum due to the fact that the field coupling function is in one case constant whereas in the other case depends on the field. We shall study two cases individually: the matter coupling will either be of the form (3) or (4).

The toy model we consider in this paper is described by the following action:

$$
S=\int d^{4} x \sqrt{-g}\left[\frac{\mathcal{R}}{2 \kappa^{2}}-g^{\mu \nu}\left(\partial_{\mu} \phi\right)\left(\partial_{\nu} \phi\right)-V(\phi)\right]+S_{V}\left(\psi_{V}, A(\phi) g_{\mu \nu}\right)+S_{I V}\left(\psi_{I V}, B(\phi) g_{\mu \nu}\right)
$$

In the context of brane worlds, the index $V$ stands here for matter on brane 1 , whereas $I V$ stands for matter on brane 2. The functions $\psi_{V}$ and $\psi_{I V}$ stand for the matter fields on each individual branes. The field $\phi$ in equation (5) takes the role of either $\varphi$ or $R$ in the last section. In the following we shall work with reduced Planck units $\kappa \equiv 1$.

From the action above, one can derive the following field equations:

$$
\begin{gathered}
G_{\mu \nu}=\left(T_{\mu \nu}^{(V)}+T_{\mu \nu}^{(I V)}+\phi_{, \mu} \phi_{, \nu}-\frac{1}{2} g_{\mu \nu}(\partial \phi)^{2}+V(\phi)\right) \\
\square \phi=-\beta_{V} T^{V}-\beta_{I V} T^{I V} \\
T_{\mu}^{(i) \nu ; \mu}=\beta_{(i)} T^{(i)} \phi^{, \nu}, \quad \text { with } i=(V, I V) .
\end{gathered}
$$

In these equations, $T$ stands for the trace of the energy-momentum tensor for each individual matter. The functions $\beta$ are given by

$$
\beta_{1}=\frac{\partial \ln A}{\partial \phi}, \quad \beta_{2}=\frac{\partial \ln B}{\partial \phi} .
$$

In the first case under consideration, the functions $\beta$ do not depend on the scalar field (as it is the case with one of the scalar field in section II) and choose $\beta$ to be the form ${ }^{4}$

$$
\text { Case I : } \quad A=B \propto \exp \left(-2 \alpha^{2} \phi /\left(1+2 \alpha^{2}\right)\right) \Rightarrow \quad \beta_{1}=-\frac{2 \alpha^{2}}{1+2 \alpha^{2}}=\beta_{2} .
$$

We have retained the main features of the brane model, i.e. $\beta_{1,2}$ is negative and bounded. In the second case (corresponding to the field $R$ in the last section), the functions $\beta$ depend on the field and we have

$$
\text { Case II : } A \propto \cosh (\phi), \quad B \propto \sinh (\phi) \Rightarrow \quad \beta_{1}=\tanh (\phi)=1 / \beta_{2} .
$$

As before we have kept the same functional dependence as in the brane models.

For a homogeneous and isotropic universe with flat spatial sections, the line element reads

$$
d s^{2}=a^{2}(\tau)\left(-d \tau^{2}+\delta_{i j} d x^{i} d x^{j}\right) .
$$

The field equations read

$$
\begin{gathered}
H^{2}=\frac{1}{3} a^{2}\left(\rho_{V}+\rho_{I V}+\frac{1}{2 a^{2}} \dot{\phi}^{2}+V(\phi)\right), \\
\ddot{\phi}+2 H \dot{\phi}+a^{2} \frac{\partial V}{\partial \phi}=-\beta_{V}\left(\rho_{V}-3 p_{V}\right) a^{2}-\beta_{I V}\left(\rho_{I V}-3 p_{I V}\right) a^{2} \\
\dot{\rho}_{(i)}+3 H\left(\rho_{(i)}+p_{(i)}\right)=\beta_{(i)}\left(\rho_{(i)}-3 p_{(i)}\right) \dot{\phi} .
\end{gathered}
$$

\footnotetext{
${ }^{4}$ Note that $\alpha$ in this expression is different from $\tilde{\alpha}$ in Section 2, because of the field redefinition.
} 
In these equations, the dot represents a derivative with respect to the conformal time $\tau$ and $H=\dot{a} / a$. Note that in equations (7) and (14) we must sum over all matter forms. In this paper we will assume that $T_{\mu \nu}^{I V}=0$. This would correspond to no matter on the second brane in the theory of the last section.

The theory above differs from General Relativity and is therefore constrained by observations. Firstly, local experiments (i.e. on earth and in the Solar System) constrain the post-Newtonian parameters $\gamma_{\mathrm{PN}}$ and $\beta_{\mathrm{PN}}$ given by

$$
\begin{aligned}
& \gamma_{\mathrm{PN}}-1=-2 \frac{\beta_{\mathrm{A} / \mathrm{B}}^{2}}{1+\beta_{\mathrm{A} / \mathrm{B}}^{2}}, \\
& \beta_{\mathrm{PN}}-1=\frac{1}{2} \frac{\beta_{\mathrm{A} / \mathrm{B}}^{2}}{\left(1+\beta_{\mathrm{A} / \mathrm{B}}^{2}\right)^{2}} \frac{d \beta_{\mathrm{A} / \mathrm{B}}}{d \phi},
\end{aligned}
$$

where $\beta_{\mathrm{A} / \mathrm{B}}$ stand for the functions $\beta_{1}$ for the cases I or II, respectively. Current constraints give [31]

$$
\left|\gamma_{\mathrm{PN}}-1\right| \leq 2 \cdot 10^{-3}, \quad\left|\beta_{\mathrm{PN}}-1\right| \leq 6 \cdot 10^{-4}
$$

Nucleosynthesis constraints limit the effective number of degrees of freedom for relativistic particles at this epoch. In general the constraints have to be worked out in detail, but there is a simple way to get a rough limit on the theory from nucleosynthesis [32]. Because the energy conservation equation (15) implies that $\rho_{\mathrm{M}} a^{3} \neq$ constant in general, the expansion rate at nucleosynthesis is different from its value in General Relativity. This leads to [32]

$$
\left(\frac{A\left(\phi_{\text {nuc }}\right)}{A\left(\phi_{0}\right)}\right)^{2} \leq 1.2
$$

Although we have derived the general equations, from now on we shall set $V(\phi)=\Lambda$, because we wish to compare the results for the perturbations with the $\Lambda \mathrm{CDM}$ model. Before we turn our attention to cosmological perturbations and their evolution, however, we briefly study the evolution of the background in the matter dominated era for the case A and B separately.

\section{Case I: The case for constant coupling parameter $\beta$}

The model in this case has similarities to the model discussed in [33], although we do not have an exponential potential for the field, and so the critical points found in [33] do not apply here. In the radiation dominated epoch, the field is almost constant, because the terms on the left-hand side on the Klein-Gordon equation (14) dominate. The constraints (18) on the post-Newtonian parameter lead to $\alpha \leq 0.1$. We can easily find the solutions to the background equations in the matter dominated epoch:

$$
\begin{aligned}
a(\tau) & =\left(\frac{\tau}{\tau_{0}}\right)^{x}, \text { with } \quad x=2-8 \alpha^{4} \\
\phi & =\phi_{0}+4 \alpha^{2} \ln \left(\frac{\tau}{\tau_{0}}\right) .
\end{aligned}
$$

Therefore, the universe expands a little slower than expected, although the correction is very small. An important point has to be made here, namely that matter does not scale like $\rho_{\text {matter }} \propto a^{-3}$, but rather like

$$
\rho_{\text {matter }} \propto a^{-3} \exp \left(\int \beta d \phi\right) .
$$

For the theory under consideration here, this implies that, for a given matter density today, the matter density in the past was greater than it would have been under standard cosmology.

Case II: The case for non-constant coupling parameter $\beta$

The low-energy effective theory now corresponds to the first model by Randall and Sundrum [16]. As we will see, there is an attractor mechanism at work, which drives the field towards small values and thereby generating small couplings between $\phi$ and matter, as it is dictated by observations on Earth and in the Solar System.

The equations are difficult to handle analytically. Assuming that $\phi$ is small and slowly varying at the onset of matter domination, one can find that 


$$
a(\tau)=\left(\frac{\tau}{\tau_{0}}\right)^{2}
$$

and that $\rho_{\text {matter }} \propto a^{-3}$. The field $\phi(\tau)$ now goes like

$$
\phi(\tau)=A \frac{\cos \left(\sqrt{\frac{39}{4}} \ln \left(\tau / \tau_{0}\right)\right)}{\left(\tau / \tau_{0}\right)^{3 / 2}}+B \frac{\sin \left(\sqrt{\frac{39}{4}} \ln \left(\tau / \tau_{0}\right)\right)}{\left(\tau / \tau_{0}\right)^{3 / 2}} .
$$

Thus, apart from oscillating behaviour, the field $\phi$ in this case decays during matter domination.

Although the solutions above are based on a crude approximation, this qualitative behaviour is found even if the field value was initially larger [22]. For large values of $\phi$, however, equation (21) is a better approximation.

Thus, although it is easy to fulfill the constraints (18) due to the cosmological attractor, the nucleosynthesis constraints give a limit on $\phi\left(\tau=\tau_{\text {nucl }}\right) \leq 0.4$.

\section{COSMOLOGICAL PERTURBATIONS AND MODULI FIELDS}

In the following we discuss the evolution of perturbations in the presence of moduli fields. This section is organized as follows: first we will write down the perturbation equations. Then we will solve them (approximately) in the matter dominated era, in order to gain some understanding about the effects of the coupling between the field and matter. Finally we discuss the solutions of the numerical computation of the spectrum of anisotropies in the CMB and the matter power spectrum.

\section{A. The Perturbation Equations in the Synchronous Gauge}

We work in the synchronous gauge (using the notations as in [34]); i.e. the perturbed line element has the form

$$
d s^{2}=a^{2}(\tau)\left(-d \tau^{2}+\left(\delta_{i j}+h_{i j}\right) d x^{i} d x^{j}\right) .
$$

After Fourier transformation, the perturbed Einstein equations read:

$$
\begin{aligned}
2 k^{2} \eta-\frac{\dot{a}}{a} \dot{h} & =a^{2} \delta T_{0}^{0} \\
\ddot{h}+2 \frac{\dot{a}}{a} \dot{h}-2 k^{2} \eta & =-a^{2} \delta T_{i}^{i},
\end{aligned}
$$

where $h$ and $\eta$ are defined by ( $\hat{\mathbf{k}}$ is the unit vector in direction of $\mathbf{k}$ )

$$
h_{i j}=\int d^{3} k e^{i \mathbf{k} \cdot \mathbf{x}}\left[\hat{\mathbf{k}}_{i} \cdot \hat{\mathbf{k}}_{j} h(\mathbf{k}, \tau)+\left(\hat{\mathbf{k}}_{i} \cdot \hat{\mathbf{k}}_{j}-\frac{1}{3} \delta_{i j}\right) 6 \eta(\mathbf{k}, \tau)\right]
$$

¿From the energy-momentum conservation equation one obtains a set of two equations for the evolution of the density contrast and the divergence of the velocity field. Defining

$$
\begin{aligned}
& (\rho+p) \theta=i k^{j} \delta T_{j}^{0} \\
& (\rho+p) \sigma=-\left(\hat{k}_{i} \cdot \hat{k}_{j}-\frac{1}{3} \delta_{i j}\right)\left(T^{i j}-\delta^{i j} T_{k}^{k} / 3\right)
\end{aligned}
$$

and writing $p_{i}=w_{(i)} \rho_{(i)}, c_{s(i)}^{2}=\partial p_{(i)} / \partial \rho_{(i)}$ and $\theta=i k^{i} v_{i}$ we find from the evolution equation for the density contrast $\delta_{(i)}=\delta \rho_{(i)} / \rho_{(i)}$

$$
\begin{aligned}
\dot{\delta}_{(i)} & =-\left(1+w_{(i)}\right)\left[\theta_{(i)}+\frac{\dot{h}}{2}\right]-3 \frac{\dot{a}}{a}\left(c_{s(i)}^{2}-w_{(i)}\right) \delta_{(i)} \\
& +\frac{\partial \beta_{i}}{\partial \phi}\left(1-3 w_{(i)}\right) \dot{\phi} \delta \phi+\beta_{i}\left(1-3 w_{(i)}\right)(\dot{\delta} \phi)-3 \dot{\phi} \beta_{i}\left(c_{s(i)}^{2}-w_{(i)}\right) \delta_{(i)} .
\end{aligned}
$$


The divergence of the velocity field $\theta_{(i)}=v^{(i) j}$ of each individual fluid evolves according to

$$
\begin{aligned}
\dot{\theta}_{(i)} & =-\frac{\dot{a}}{a}\left(1-3 w_{(i)}\right) \theta_{(i)}-\frac{\dot{w}_{(i)}}{1+w_{(i)}} \theta_{(i)}+\frac{c_{s(i)}^{2}}{1+w_{(i)}} k^{2} \delta-k^{2} \sigma_{(i)} \\
& +\beta_{i} \frac{1-3 w_{(i)}}{1+w_{(i)}} k^{2} \delta \phi-\beta_{i}\left(1-3 w_{(i)}\right) \dot{\phi} \theta_{(i)} .
\end{aligned}
$$

It is important to note that these equations reduce to those of ordinary General Relativity, if the fluid under consideration has an radiation-like equation of state, i.e. if $w_{(i)}=1 / 3$. This means that the equations governing the dynamics of photons and relativistic neutrinos are those found in General Relativity. Important changes occur in the equations for CDM and baryons:

\section{Cold Dark Matter:}

In this case, we have $w=0=c_{s}$ and vanishing anisotropic stress. Thus

$$
\begin{gathered}
\dot{\delta}_{c}=-\left[\theta_{c}+\frac{\dot{h}}{2}\right]+\frac{\partial \beta_{c}}{\partial \phi} \dot{\phi} \delta \phi+\beta_{c}(\dot{\delta \phi}) \\
\dot{\theta}_{c}=-\frac{\dot{a}}{a} \theta_{c}+\beta_{c} k^{2} \delta \phi-\beta_{c} \dot{\phi} \theta_{c}
\end{gathered}
$$

\section{Baryons:}

In this case, we have $w=0=c_{s}$ and vanishing anisotropic stress (a very good approximation after neutrino decoupling [34]). As in [34], we do not neglect the quantity $c_{s}^{2} k^{2} \delta$, which becomes important on small length scales and thus large $k$. We also have to add the terms describing the momentum transfer between photons and baryons due to Thomson scattering. Therefore, we have

$$
\begin{gathered}
\dot{\delta}_{b}=-\left[\theta_{b}+\frac{\dot{h}}{2}\right]+\frac{\partial \beta_{b}}{\partial \phi} \dot{\phi} \delta \phi+\beta_{b}(\dot{\delta \phi}) \\
\dot{\theta}_{b}=-\frac{\dot{a}}{a} \theta_{b}+c_{s}^{2} k^{2} \delta_{b}+\frac{4 \rho_{\gamma}}{3 \rho_{b}} a_{e} \sigma_{T}\left(\theta_{\gamma}-\theta_{b}\right)+\beta_{b} k^{2} \delta \phi-\beta_{b} \dot{\phi} \theta_{b}
\end{gathered}
$$

where $n_{e}$ is the number density of free electrons and $\sigma_{T}$ the Thomson cross section.

The only new terms compared to General Relativity are the ones proportional to $\beta$ and its derivative. Note that we have to add an evolution equation for $\theta_{c}$ : while we can still set $\theta_{c}=0$ initially (in order to fix the synchronous gauge completely), in General Relativity, where there is no extra field, it stays zero; however, fluctuations in $\phi$ are a source and therefore, in the theory we consider, it evolves according to the above equation.

Finally, the equation for the fluctuations in $\phi$ evolve according to

$$
(\ddot{\delta \phi})+2 \frac{\dot{a}}{a}(\dot{\delta \phi})+\left(k^{2}+a^{2} \frac{\partial^{2} V}{\partial \phi^{2}}\right) \delta \phi+\frac{1}{2} \dot{h} \dot{\phi}=-\left(\beta_{c} \rho_{c} \delta_{c}+\beta_{b} \rho_{b} \delta_{b}+\frac{\partial \beta_{b}}{\partial \phi} \rho_{b}(\delta \phi)+\frac{\partial \beta_{c}}{\partial \phi} \rho_{c}(\delta \phi)\right) a^{2}
$$

These equations are quite general. Again, we are assuming $V=\Lambda=$ const.

\section{B. Solutions to the Perturbation Equations in the Matter Dominated Era}

Before we give the results for the CMB anisotropies and matter power spectrum, we try to gain some analytical insight from the perturbation equations in the matter dominated epoch on sub-horizon scales. We neglect radiation and the baryons, taking only into account CDM and the scalar field in this regime. Furthermore, we consider only the case for the field with constant coupling, which is tractable analytically.

Taking the derivative of equation (32) and making use of equations (25), (26), (33) and the Friedmann equation, one gets 


$$
\ddot{\delta}_{c}+H \dot{\delta}_{c}-\frac{3}{2} H^{2} \delta_{c}=2 \alpha^{2} k^{2} \delta \phi+2 \dot{\phi}(\dot{\delta \phi})-2 \alpha^{2}(H(\dot{\delta \phi})+(\ddot{\delta \phi}))-2 \alpha^{2} \dot{\phi} \theta_{c} .
$$

Using the perturbed Klein-Gordon equation, ignoring oscillations in $\delta \phi$ and inserting (32) one obtains

$$
\ddot{\delta}_{c}+\left(H-2 \alpha^{2} \dot{\phi}\right) \dot{\delta}_{c}-\frac{3}{2} H^{2}\left(1+8 \alpha^{4}\right) \delta_{c}=0 .
$$

Assuming $a \propto \tau^{x}$, using the background solution equation (20) and making the Ansatz $\delta_{c} \propto \tau^{m}$, one gets up to $\mathcal{O}\left(\alpha^{4}\right)$

$$
m=2+\frac{32}{5} \alpha^{4}
$$

for the growing mode. Therefore, compared to the $\Lambda$ CDM model, due to the non-vanishing coupling perturbations in CDM grow faster than the scale factor and the transfer function is on small scales bent towards larger values. We have confirmed this numerically.

\section{CMB Anisotropies and the Matter Power Spectrum}

In the following we will discuss the spectrum of anisotropies in the CMB and the matter power spectrum. It is customary to expand the temperature anisotropy measured in a given direction $\mathbf{n}$ in spherical harmonics according to

$$
\frac{\Delta T}{T}(\mathbf{n})=\sum_{l m} a_{l m} Y_{l m}(\mathbf{n}) .
$$

We assume that the density fluctuations are Gaussian random variables, as predicted by the inflationary scenario. The statistics of the fluctuations are completely specified by the power spectrum $C_{l}$, defined as

$$
\left\langle a_{l m} a_{l^{\prime} m^{\prime}}^{*}\right\rangle \equiv C_{l} \delta_{l l^{\prime}} \delta_{m m^{\prime}} .
$$

Let us briefly summarize how the $C_{l}$ s depend on the perturbation variables and how they are calculated (see [35], [36] and [3] for excellent detailed reviews on the physics of CMB anisotropies, [37]- [40] are shorter reviews). The reader familiar with CMB anisotropies can skip this part. To illustrate the essential steps involved in calculating the anisotropy power spectrum, we neglect the polarization states of the photons. However, when we present the results of the calculations, the polarization states have been taken into account.

At very early times the universe was radiation dominated and the temperature of the universe was so high, that photons and baryons formed a plasma. At these high temperatures, photons and baryons are tightly coupled. On scales less than the horizon the fluid undergoes acoustic oscillations due to the interplay of gravity, which tries to compress overdense regions, and the photon pressure, which, when high enough, acts against the force of gravity. When the temperature dropped below $4000 \mathrm{~K}$, electrons and nuclei were able to combine to atoms (mainly hydrogen and helium) and the mean free path of the photons became larger than the Hubble horizon. Around that time, the photons completely decoupled from matter. On their path, photons may experience energy loss due to time-changing gravitational fields (the so-called integrated Sachs-Wolfe effect, or ISW). Additionally, photons may interact with reionized gas (reionization might happen due to early star formation).

In order to follow the perturbations in the photons correctly, one has to go beyond the fluid description and study the evolution of the photon distribution function $f_{\gamma}$ in phase space, i.e. one has to solve the Boltzmann equation. In general, $f_{\gamma}$ is a function of the comoving position $\mathbf{x}$, the photon momentum $\mathbf{p}$ and conformal time $\tau$. To first order we write $f_{\gamma}=f_{0}+\delta f_{\gamma}$, where $f_{0}$ is the unperturbed photon distribution. The brightness function $\Delta_{T}(\mathbf{x}, \mathbf{n}, \tau)$ is defined as

$$
\delta f_{\gamma}(\mathbf{x}, \mathbf{p}, \tau)=\left(\frac{\bar{T}}{4} \frac{\partial f_{0}}{\partial \bar{T}}\right) \Delta_{T}(\mathbf{x}, \mathbf{n}, \tau)
$$

where $\bar{T}$ is the unperturbed temperature of the photon gas (i.e. of the background) and $\mathbf{n}$ is a unit vector in the direction of the photon momentum p. One can now formulate a perturbed version of the Boltzmann equation, describing the evolution of $\Delta_{T}$. However, it is more useful to perform a Fourier transform of $\Delta_{T}(\mathbf{x}, \mathbf{n}, \tau)$ and expand the Fourier coefficients $\Delta_{T}(\mathbf{k}, \mathbf{n}, \tau)$ in Legendre polynomials ( $\hat{\mathbf{k}}$ is a unit vector in direction of $\left.\mathbf{k}\right)$ :

$$
\Delta_{T}(\mathbf{k}, \mathbf{n}, \tau)=\sum_{l=0}^{\infty}(-i)^{l}(2 l+1) \Delta_{T l}(k, \tau) P_{l}(\hat{\mathbf{k}} \cdot \mathbf{n}) .
$$


It can be shown that the density contrast in the photon energy density, $\delta_{\gamma}$, the divergence of the photon velocity field, $\theta_{\gamma}$ and the photon anisotropic stress, $\sigma_{\gamma}$, are related to $\Delta_{T l}$ by

$$
\delta_{\gamma}=\Delta_{T 0}, \quad \theta_{\gamma}=\frac{3}{4} k \Delta_{T 1}, \quad \sigma_{\gamma}=\frac{1}{2} \Delta_{T 2}
$$

In terms of the moments $\Delta_{T l}$, the Boltzmann equation becomes a hierarchy of equations. Using equation (44), the evolution equations are given by

$$
\begin{aligned}
\dot{\delta}_{\gamma} & =-\frac{4}{3} \theta_{\gamma}-\frac{2}{3} \dot{h}, \\
\dot{\theta}_{\gamma} & =k^{2}\left(\frac{1}{4} \delta_{\gamma}-\sigma_{\gamma}\right) a n_{e} \sigma_{T}\left(\theta_{b}-\theta_{\gamma}\right), \\
2 \dot{\sigma}_{\gamma} & =\frac{8}{15} \theta_{\gamma}-\frac{3}{5} k \Delta_{T 3}+\frac{4}{15} \dot{h}+\frac{8}{5} \dot{\eta}-\frac{9}{5} a n_{e} \sigma_{T} \sigma_{\gamma} \\
\dot{\Delta}_{T l} & =\frac{k}{2 l+1}\left[l \Delta_{T(l-1)}-(l+1) \Delta_{T(l+1)}\right]-a n_{e} \sigma_{T} \Delta_{T l}, \quad(l \geq 3)
\end{aligned}
$$

Furthermore, it can be shown that the quantity $C_{l}$, defined in equation (41), is related to the brightness function $\Delta_{T l}$ by

$$
C_{l}=4 \pi \int \frac{d k}{k}\left|\Delta_{T l}\left(k, \tau_{0}\right)\right|^{2}
$$

It is straightforward to include polarization. Due to Thomson scattering, photons are linearly polarized in the plane perpendicular to $\mathbf{n}$. The collision terms in the Boltzmann equation depend on the polarization state. In order to follow the evolution in phase space, one defines a total distribution function $f_{\gamma}$, which includes all polarization states and another distribution function, $g_{\gamma}$, which is defined as the difference of the distribution functions for the individual polarized photons. Defining the temperature brightness function $\Delta_{T}$ and the polarization brightness function $\Delta_{P}$ similarly to the above procedure, one can show that $\Delta_{T}$ obeys the equation (45)-(48), but equation (47) has to be changed to

$$
2 \dot{\sigma}_{\gamma}=\frac{8}{15} \theta_{\gamma}-\frac{3}{5} k \Delta_{T 3}+\frac{4}{15} \dot{h}+\frac{8}{5} \dot{\eta}-\frac{9}{5} a n_{e} \sigma_{T} \sigma_{\gamma}+\frac{1}{10} a n_{e} \sigma_{T}\left(\Delta_{P 0}-\Delta_{P 2}\right),
$$

because the collision term in the Boltzmann equation depends on the polarization state. Additionally, one obtains a hierarchy for the functions $\Delta_{P l}$ :

$$
\dot{\Delta}_{P l}=\frac{k}{2 l+1}\left[l \Delta_{P(l-1)}-(l+1) \Delta_{P(l+1)}\right]-a n_{e} \sigma_{T}\left[\Delta_{P l}+\frac{1}{2}\left(\Delta_{T 2}+\Delta_{P 0}+\Delta_{P 2}\right)\left(\delta_{0 l}+\frac{\delta_{2 l}}{5}\right)\right],
$$

where $\delta_{i l}$ in the last term stands for the Kronecker delta.

The CMB anisotropy power spectrum $C_{l}$ is still given by equation (49). Thus, in order to calculate the CMB power spectrum $C_{l}$ (as well as the polarization power spectrum and the temperature/polarization cross correlation spectrum), one has to solve a large set of differential equations (the equations for baryons, CDM and the scalar field presented in section IV A and the hierarchy above). In [41] solutions to the Boltzmann equation are found. Here we use an existing code [42] and make the appropriate changes to the evolution of background and perturbation variables to incorporate the above theory.

\section{Constant Coupling}

In order to calculate the CMB power spectrum, we make changes to an existing code [42], whose method is based on [43]. As described in section IV A we have to make changes in the equations for CDM and baryons and have to include the modified Klein-Gordon equation for the scalar field. We assume a scale-invariant initial power spectrum and adiabatic initial conditions.

What are the expected effects of the non-vanishing coupling? There are two effects, which come in when $\alpha$ differs from zero. Firstly, as mentioned in section III, the dependence of the matter density (both for baryons and CDM) on the scale factor is no longer given by $\rho_{\text {matter }} \propto a^{-3}$, but rather by equation (21). Therefore, relative to the $\Lambda$ CDM 
model the density of matter (and in particular of baryons) is larger at decoupling (we remind the reader that the models are normalized such that their matter density is equal at the present epoch). Hence, the amplitudes of the peaks are larger, since a larger baryon content implies larger amplitudes of the peaks.

Additionally, there are changes to the sound speed $c_{s}^{2}=\dot{p}_{\gamma} /\left(\dot{\rho}_{\mathrm{b}}+\dot{\rho}_{\gamma}\right)$. The sound speed decreases with increasing $\alpha$. This effects the peak separation, which is proportional to [35]

$$
\delta l=\frac{\chi\left(\tau_{0}-\tau_{\mathrm{dec}}\right)}{D_{s}}
$$

where $\chi\left(\tau_{0}-\tau_{\mathrm{dec}}\right)$ is the angular diameter distance to the surface of last scattering and $D_{s}=\int^{\tau_{\mathrm{dec}}} c_{s} d \tau$ is the sound horizon. Aside from having an effect on $c_{s}$, the presence of the scalar field does also affect the distance to the last scattering surface. Let us estimate the position of the first peak, given by $l_{0}=\pi \chi\left(\tau_{0}-\tau_{\text {dec }}\right) / D_{s}$. It is simple to calculate $\chi\left(\tau_{0}-\tau_{\mathrm{dec}}\right)$ :

$$
\chi\left(\tau_{0}-\tau_{\mathrm{dec}}\right)=\int_{\tau_{\mathrm{dec}}}^{\tau_{0}} d \tau \approx \frac{2}{H_{0}} \frac{1}{1+C}
$$

where $C=2 \alpha^{4} /\left(1-8 \alpha^{2}\right)\left(1+2 \alpha^{2}\right)$. Putting $c_{s}^{2} \approx 1 / 3$ and neglecting the radiation dominated epoch, one finds

$$
D_{s} \approx \frac{1}{3} \frac{1}{H_{0}} \frac{2}{1+C} a_{\mathrm{dec}}^{\frac{1}{2}+\frac{C}{2}} .
$$

Therefore, the position of the peak is given by (note that we have $a\left(\tau_{0}\right)=1$ )

$$
l_{0}=\tilde{l}_{0} a_{\mathrm{dec}}^{-C / 2},
$$

where $\tilde{l}_{0} \approx 200$ is the position of the peak for the flat model with no coupling. Therefore, the peak moves towards larger values (i.e. to the right in the spectrum).

To reach this conclusion we have assumed that $c_{s} \approx 1 / 3$, which is a crude assumption. Note, however, that if one takes the baryons into account the $c_{s}$ is smaller and hence, the sound horizon $D_{s}$ is smaller. Thus, $l_{0}$ is if anything somewhat larger than the value given in equation (55).

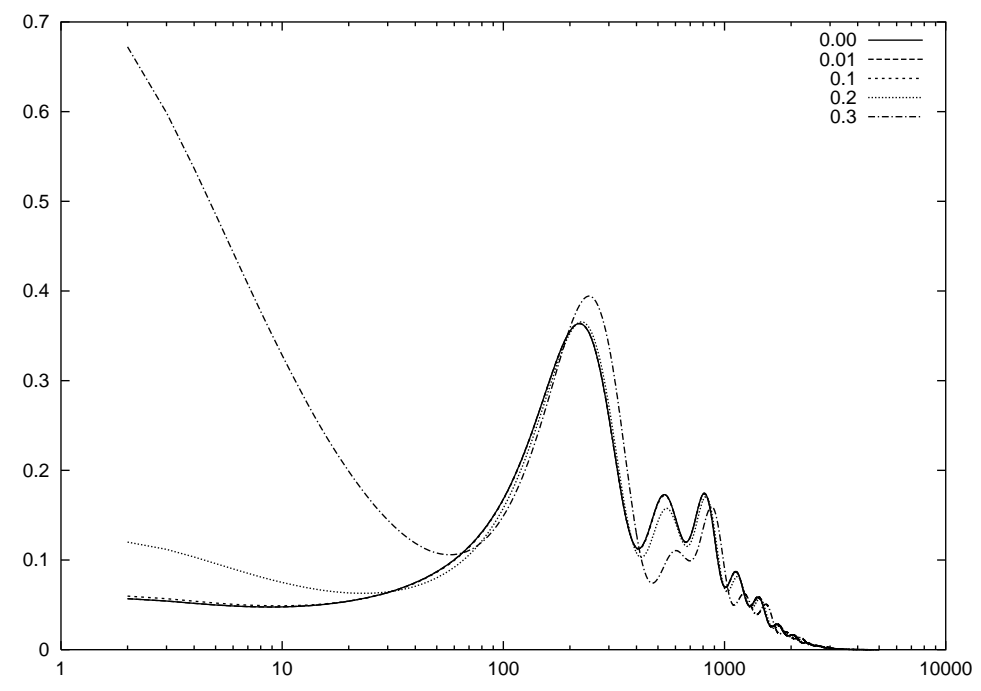

FIG. 2. The temperature anisotropy power spectrum, $l(l+1) C_{l} / 2 \pi$, for the constant coupling case: the values in the legend are the values of $\alpha$.

The second important change when the coupling is non-vanishing is that there is a larger contribution from the integrated Sachs-Wolfe effect. Even in the matter dominated era the gravitational potential changes with time, because the matter density contrast no longer grows like $\delta(\tau) \propto a(\tau)$, as discussed in the last section. As the timeevolution of the scale factor is altered in the presence of the scalar field, the duration of the recombination era is affected, too. 
In figure 2 we plot the results for the temperature anisotropy power spectra. We assume an initial power spectrum with equal power on all scales. It can clearly be seen that the both the amplitude and the separation of the peaks is affected with increasing coupling. Also, on large scales (low $l$ ) one can see the additional power due to the integrated Sachs-Wolfe effect.

In order to compare the theory to observations, we normalize the curves appropriately. Usually this done at large angular scales, using the COBE data as reference. The curves normalized to COBE are plotted in figure 3. Clearly, the normalized curves show less power on small scales because of the enhanced integrated Sachs-Wolfe effect in models with non-vanishing coupling. On larger scales (small multipole number $l$ ) one clearly sees a boost of power. Additionally, the normalized matter power spectra for models with non-vanishing coupling are below the $\Lambda$ CDM curve.

For completeness we plot the E-type polarization spectra and the cross correlation between temperature and E-type polarization in figure 4 . One can clearly see that in both spectra on small scales the power is suppressed when $\alpha$ is increased.
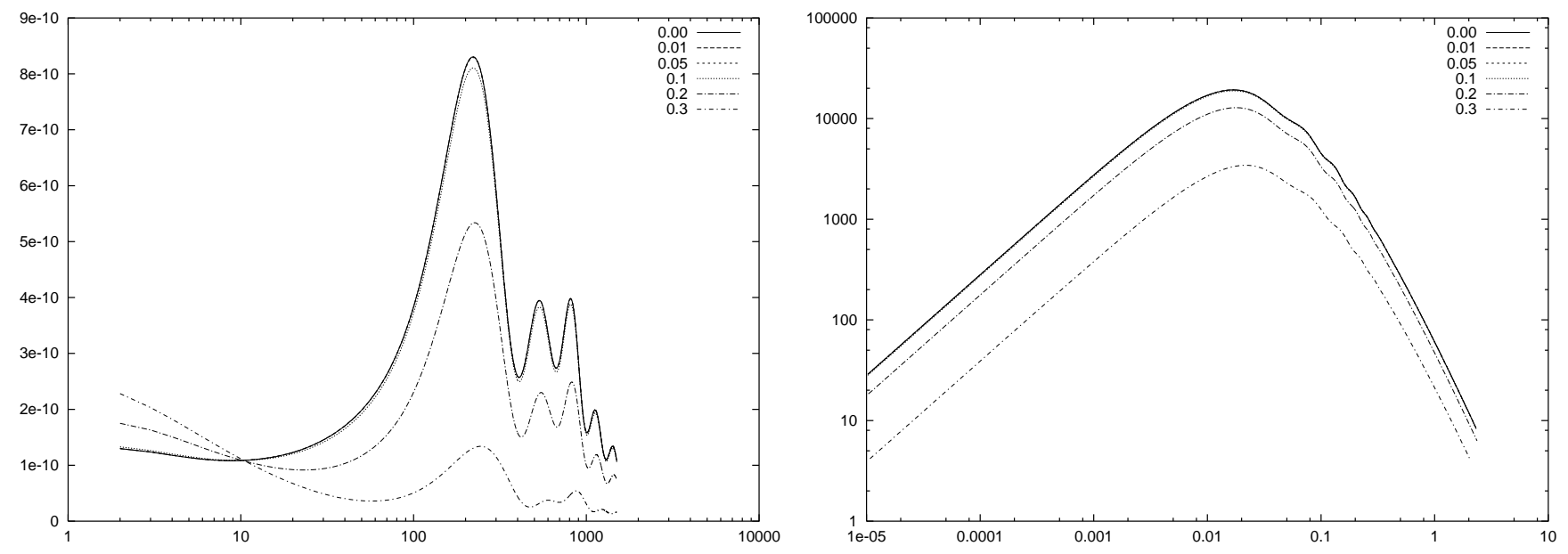

FIG. 3. COBE-normalized temperature anisotropy $l(l+1) C_{l} / 2 \pi$ (left panel) and matter power spectrum (right panel) for the case of constant coupling. On small scales the COBE-normalized spectra are below the predictions for vanishing coupling due to the enhanced ISW.
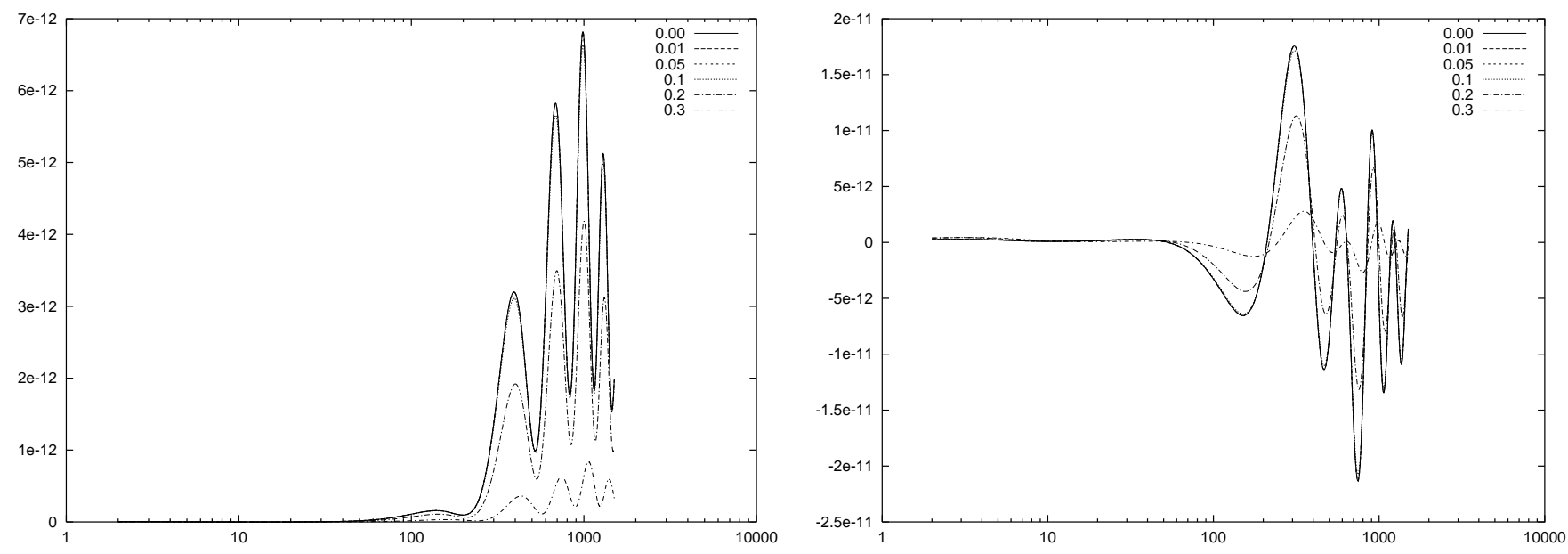

FIG. 4. COBE-normalized E-mode polarization anisotropy (left panel) and TE cross-correlation (right panel) for the case of constant coupling. 

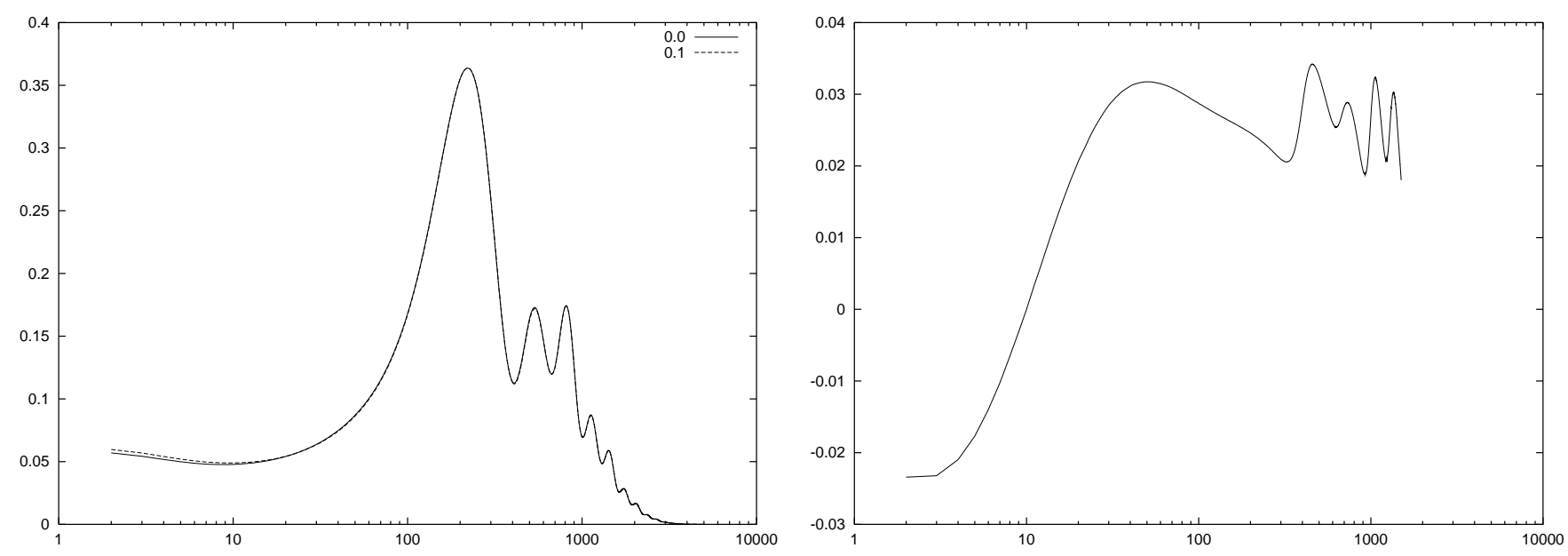

FIG. 5. CMB anisotropy spectra (left panel) for the constant-coupling case, with $\alpha$ taking the extreme value permitted by nucleosynthesis, with a reference curve from the $\Lambda \mathrm{CDM}$ model; the right panel shows the relative discrepancy. Although the differences are around 3 percent at high multipoles, a different choice of normalization - normalizing to COBE at $l=800$, say - would move all the observed discrepancies into the low-multipole region, where cosmic variance dominates.

In figure 5 we plot the anisotropy spectra for the $\Lambda$ CDM model and the model with the maximal value of $\alpha$ allowed by nucleosynthesis $(\alpha=0.1)$. One can see that the COBE normalized curves differ from each other by a few percent on small angular scales.

\section{Non-constant Coupling}

In this case, where the field $\phi$ corresponds to the $R$ field in equation 1 , the coupling function now decays. But also here, the baryon density is larger at decoupling compared to the $\Lambda$ CDM, which leaves a similar effect on the peak position and amplitudes as in the case of constant coupling.

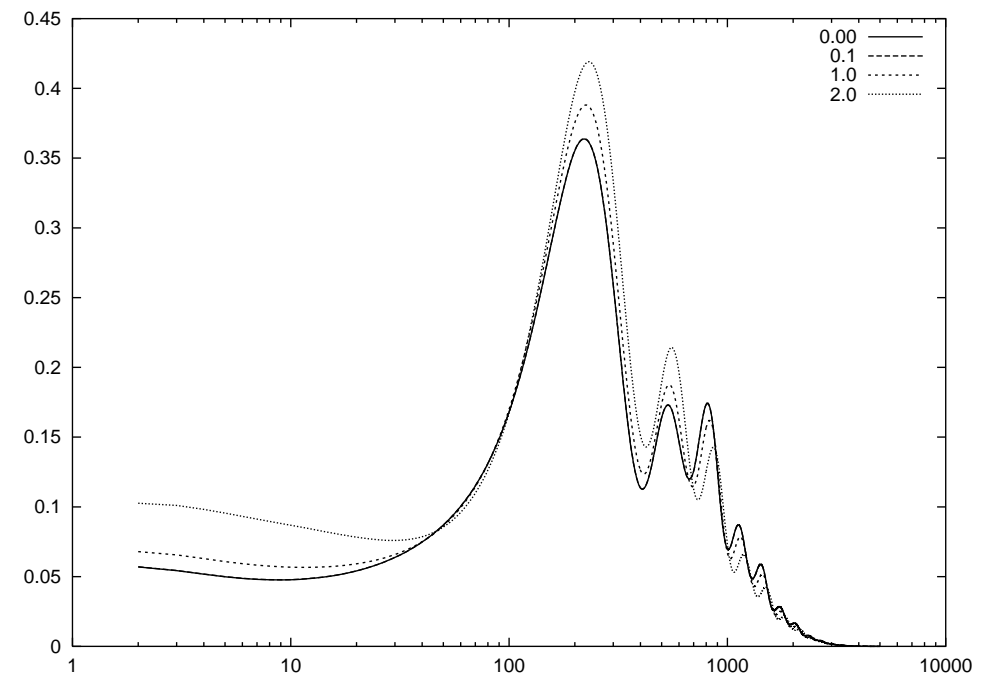

FIG. 6. The temperature anisotropy power spectrum, $l(l+1) C_{l} / 2 \pi$, for the field-dependent coupling case: the values in the legend are for the initial values of the scalar field $\phi$.

However, well inside the matter dominated epoch, the coupling becomes very small, so that the density contrast 
grows essentially like $a(\tau)$ from that time on. Therefore, the ISW effect is not as pronounced as in the case of constant coupling. Only if $\phi$ was initially very large will the field have not evolved to small values by today; consequently, for the field-dependent coupling case, only for large initial field values will the effect on the ISW become more apparent (see figure 6).
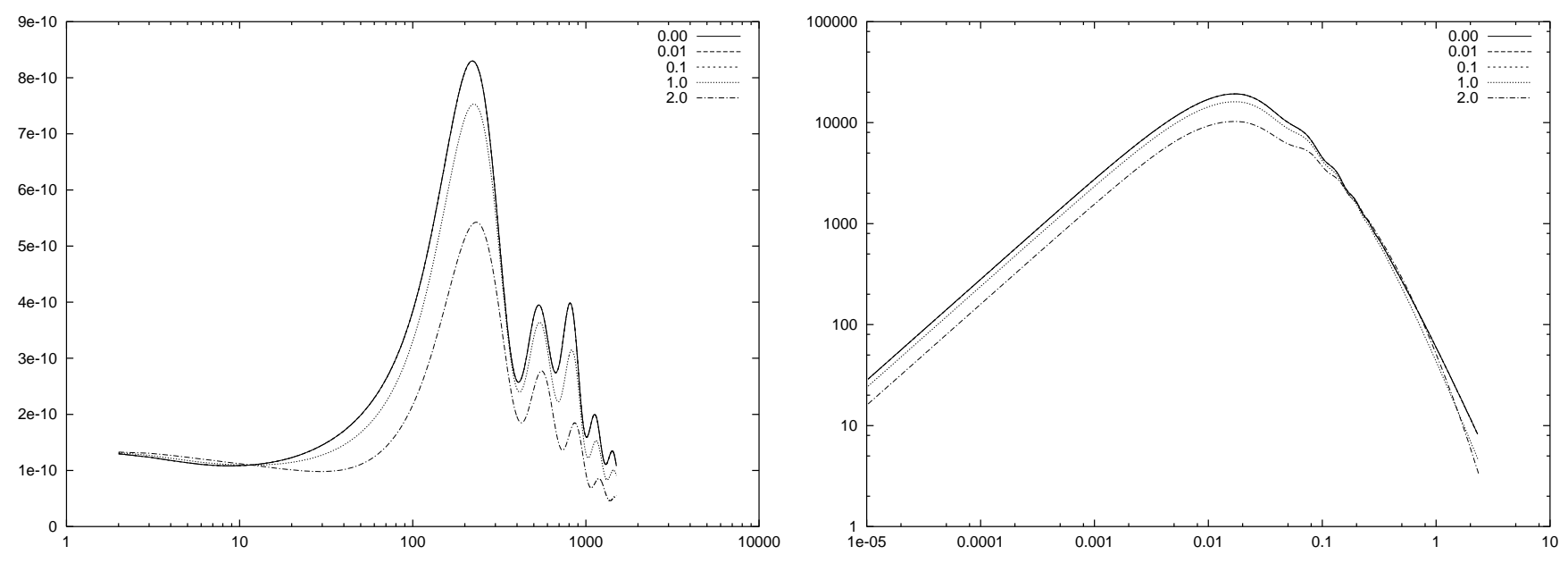

FIG. 7. COBE-normalized temperature anisotropy $l(l+1) C_{l} / 2 \pi$ (left panel) and matter power spectrum (right panel) for the case of field-dependent coupling. Similar to the case in figure 3, on small scales the COBE-normalized spectra are below the predictions for vanishing coupling.
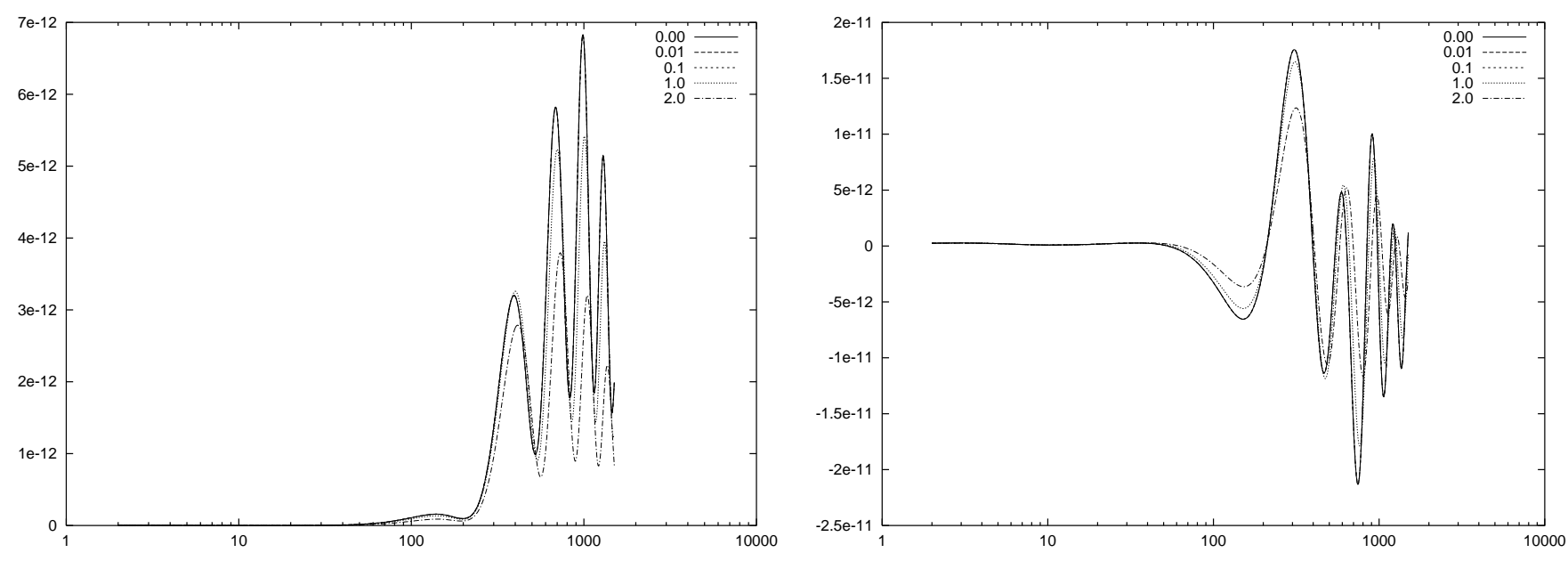

FIG. 8. COBE-normalized E-mode polarization anisotropy (left panel) and TE cross-correlation (right panel) for the case of field-dependent coupling.

The normalized anisotropy and matter power spectra are shown in figure 7, whereas the normalized E-mode polarization anisotropy and the TE-cross-correlation are shown in figure 8. Increasing the initial value of the field implies that the power in both the E-polarization spectrum and the cross correlation spectrum between temperature and $\mathrm{E}$-polarization is suppressed on small scales.

The initial field values in figures (6) and (7) have been used without taking into account constraints from nucleosynthesis (19), which implies that $\phi_{\text {nuc }}<0.4$. In figure 9 we plot the cases for $\phi_{\text {init }}=0.4$ and the $\Lambda$ CDM curve. One can see that there are differences in the predictions for the temperature anisotropy as large as 6 percent at small angular scales and 3 percent on degree scale. This is potentially distinguishable with WMAP and the Planck Surveyor. 

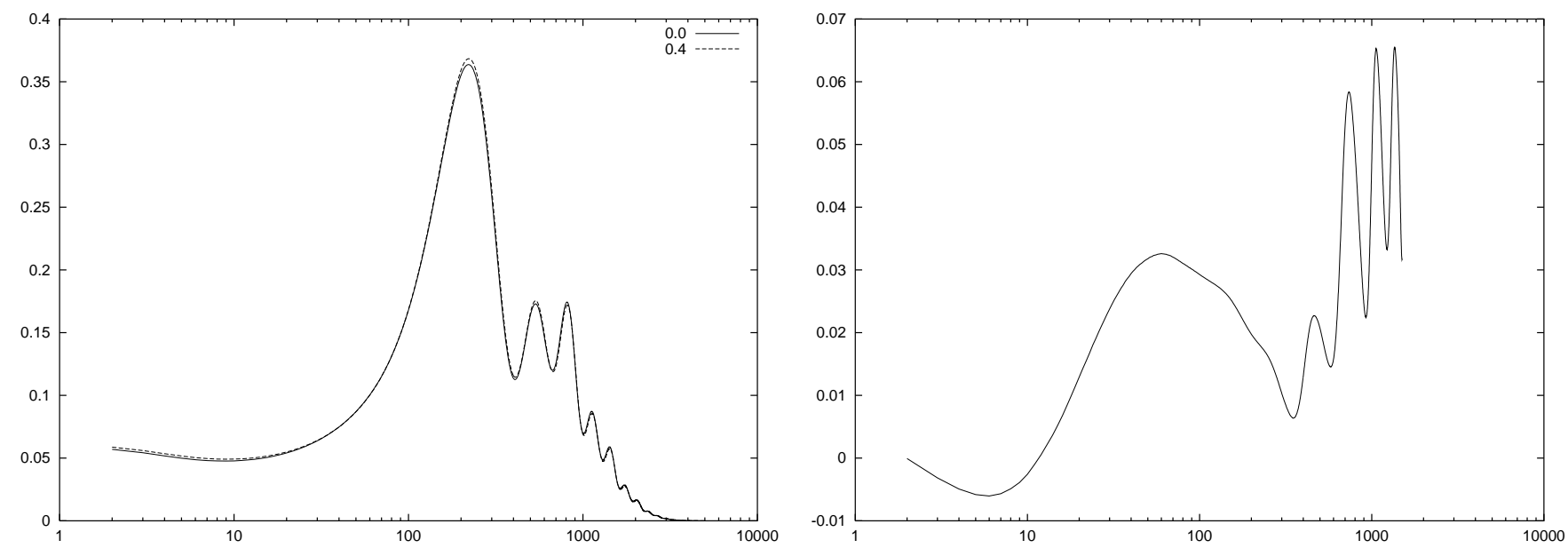

FIG. 9. CMB anisotropy spectra (left panel) for the case of an initial value $\left(\phi_{\text {ini }}=0.4\right)$ allowed by nucleosynthesis. The right panel shows the relative error between the $\Lambda \mathrm{CDM}$ and the model with $\phi_{\text {ini }}=0.4$. For $l \approx 100$ the difference between the model is as large as 3 percent and gets larger at higher $l$ (smaller angular scales).

We would like to point out that constraints on $\phi$ become stronger, once the CMB is included. In all cases plotted in the figures, the field fulfills the constraints today. However, a large field value in the early universe modifies the predictions for the temperature and polarization power spectra (as well as the matter power spectra). Hence, the CMB provides vital complementary information to the local experiments.

\section{CONCLUSION}

In this paper we have investigated the implications of time-varying extra dimensions for CMB anisotropies and large scale structure formation. In theories with extra dimensions, certain scalar fields, so called 'moduli fields', appear in the low energy effective theory coupling directly to matter fields. We have investigated in particular the couplings appearing in a five-dimensional model based on the brane world idea. The model contains two branes which are the boundaries of the higher-dimensional spacetime, with, in general, four-dimensional matter fields confined on these branes. Additionally, we allowed for a scalar field propagating in the extra-dimensional spacetime. At low energies the dynamics of this system is described by a bi-scalar-tensor theory. One of the scalar fields has a constant (i.e. field- and coordinate-independent) coupling to the matter fields, whereas in the case of the second field the coupling function is field dependent.

We have investigated the effects of the individual fields on the CMB anisotropies, the cross-correlation between temperature and polarization of the CMB and the matter power spectrum. Due to the coupling, both the amplitude and the positions of the peaks are affected. The spectra normalized to COBE lie below the spectra of the reference $\Lambda \mathrm{CDM}$ model on small scales.

The results presented in section IV imply that, for the brane world model presented in section II, potentially there is a measurable effect of both fields on CMB. If it turns out that the second field needs to be already quite small during the radiation dominated epoch, then some mechanism has to drive it to these small values in the very early universe. This can happen, for example, if there is some matter on the second brane. In this case, the field $R$ is driven even faster to zero, so that it might be small at decoupling. Note, however, that in this case the evolution of perturbations are affected in the radiation dominated epoch.

Clearly, the CMB anisotropies as well as the polarization provide complementary information about time-evolution of the extra dimensions. Together with other tests, such as gravity experiments in the solar system and/or on earth and tests of time-varying gauge couplings and primordial nucleosynthesis, these cosmological considerations strongly constrain any model involving extra dimensions. For example in the case of the model considered here, the field with constant coupling is already constrained by local experiments such that its effects on the CMB are negligible. For the second field, however, we have seen that although its effect is constrained to be minimal today, it can have a big impact on the CMB anisotropies. 
There is another important point not addressed in this paper: we have not discussed the time evolution of KaluzaKlein excitations and their influence on the CMB. They might still be present in the radiation dominated epoch, thereby affecting the evolution of perturbations at least initially. Their presence could imply, for example, that the initial perturbations are not purely adiabatic. The details would depend on the mechanism which produces the primordial fluctuations, such as inflation or the cyclic model, see e.g. [44] and [45]. To address this important issue one has to go beyond the moduli space approximation (and in fact beyond the lowest order in the method used in [24], [25] and [27]).

In this paper we have investigated the effects of moduli fields on the CMB motivated from a certain class of brane world theories. There are other brane world models, in which the theory at low energies cannot be described by an action of the form (1). For example, in the models of [46]- [48], gravity becomes five-dimensional at large distances. We expect that some of the conclusions drawn in this paper do not hold for such models. Nevertheless, the CMB gives useful constraints on these models, too [49].

Acknowledgements: We are grateful to Constantinos Skordis and Domenico Tocchini-Valentini for useful discussions and comments and to Anthony Lewis for providing his CAMB quintessence code. We acknowledge support from the Anglo-French alliance exchange programme. This work is supported in part by PPARC. P.B. is partially fundedd by the RTN European programme HPRN-CT-2000-00148.

[1] A.R. Liddle and D.H. Lyth, Cosmological Inflation and Large Scale Structure, Cambridge University Press (2000)

[2] J. Peacock, Cosmological Physics, Cambridge University Press (1999)

[3] S. Dodelson, Modern Cosmology, Academic Press (2003)

[4] C.L. Bennett, M. Halpern, G. Hinshaw, N. Jarosik, A. Kogut, M. Limon, S.S. Meyer, L. Page, D.N. Spergel, G.S. Tucker, E. Wollack, E.L. Wright, C. Barnes, M.R. Greason, R.S. Hill, E. Komatsu, M.R. Nolta, N. Odegard, H. Peiris, L. Verde and J.L. Weiland, astro-ph/0302207

[5] Y. Fujii and K-I Maeda, The Scalar-Tensor Theory of Gravitation, Cambridge University Press (2003)

[6] X-L Chen and M. Kamionkowski, Phys. Rev. D 60, 104036 (1999)

[7] A. Riazuelo and J-P Uzan, Phys. Rev. D 66, 023525 (2002)

[8] R. Nagata, T. Chiba and N. Sugiyama, Phys. Rev. D 66, 103510 (2002)

[9] L. Amendola, C. Quercellini, D. Tocchini-Valentini and A. Pasqui, ApJL 583, L53 (2003)

[10] R. Bean, Phys. Rev. D64, 123516 (2001)

[11] O. Zahn and M. Zaldarriaga, Phys. Rev. D 67, 063002 (2003)

[12] J. Polchinski, String Theory, Cambridge University Press (1999)

[13] Ph. Brax and C. van de Bruck, Class. Quant. Grav. 20, R201 (2003)

[14] D. Langlois, Prog. Theor. Phys. Suppl.148, 181 (2003)

[15] R. Maartens, Prog. Theor. Phys. Suppl.148, 213 (2003)

[16] L. Randall and R. Sundrum, Phys. Rev. Lett.83, 3370 (1999)

[17] T. Damour and K. Nordtvedt, Phys. Rev. D 48, 3436 (1993)

[18] A. Lukas, B.A. Ovrut, K.S. Stelle and D. Waldram, Nucl. Phys. B 552, 246 (1999)

[19] P. Binetruy, C. Deffayet and D. Langlois, Nucl. Phys. B 565, 269 (2000)

[20] A. Lukas, B.A. Ovrut and D. Waldram, Phys. Rev. D 61, 023506 (2000)

[21] J.M. Cline and J. Vinet, JHEP 0202, 042 (2002)

[22] Ph. Brax, C. van de Bruck, A.-C. Davis and C.S. Rhodes, Phys. Rev. D 67, 023512 (2003)

[23] Ph. Brax and A.-C. Davis, Phys. Lett. B 497, 289 (2001)

[24] S. Kanno and J. Soda, Phys. Rev. D 66083506 (2002)

[25] S. Kanno and J. Soda, hep-th/0303203

[26] S. Kobayashi and K. Koyama, JHEP 0212:056 (2002)

[27] T. Shiromizu, K. Koyama and K. Takahashi, Phys. Rev. D 67, 084022 (2003)

[28] K. Koyama, astro-ph/0303108

[29] L. Randall and R. Sundrum, Phys. Rev. Lett. 83, 4690 (1999)

[30] V. Faraoni, E. Gunzig and P. Nardone, Fund. Cosmic Phys. 20, 121 (1999)

[31] C. Will, Living Rev.Rel. 44 (2001)

[32] N. Bartolo and M. Pietroni, Phys. Rev. D 61023518 (1999)

[33] L. Amendola, Phys. Rev. D 62043511 (2000) 
[34] C-P Ma and E. Bertschinger, ApJ 455, 7 (1995)

[35] R. Durrer, J. Phys. Stud. 5, 177 (2001)

[36] W. Hu and S. Dodelson, Ann. Rev. Astron. Astrophys. 40, 171 (2002)

[37] R. Durrer, astro-ph/0109274

[38] M. Zaldarriaga, astro-ph/0305272

[39] W. Hu, Annals Phys. 303203 (2003)

[40] E. Wright, astro-ph/0305591

[41] W. Hu and N. Sugiyama, ApJ 444, 489 (1995)

[42] A. Lewis, A. Challinor and A. Lasenby, ApJ 538, 473 (2000)

[43] U. Seljak and M. Zaldarriaga, ApJ 469, 437 (1996)

[44] P.J. Steinhardt and N. Turok, Phys. Rev. D 65126003 (2002)

[45] S. Gratton, J. Khoury, P.J. Steinhardt and N. Turok, astro-ph/0301395

[46] R. Gregory, V.A. Rubakov and S.M. Sibiryakov, Phys. Rev. Lett. 845928 (2000)

[47] G.R. Dvali, G. Gabadadze and M. Porrati, Phys. Lett. B 485208 (2000)

[48] G.R. Dvali, G. Gabadadze and M. Porrati, Phys. Lett. B 484112 (2000)

[49] P. Binetruy and J. Silk, Phys. Rev. Lett. 87031102 (2001) 